\title{
Dietary omega-3 polyunsaturated fatty acid and alpha-linolenic acid are associated with physical capacity measure but not muscle mass in older women $65-72$ years
}

\author{
Masoud Isanejad ${ }^{1,2} \mathbb{D} \cdot$ Behnam Tajik $^{3} \cdot$ Anne McArdle $^{1} \cdot$ Marjo Tuppurainen $^{4} \cdot$ Joonas Sirola $^{3} \cdot$ Heikki Kröger $^{3}$. \\ Toni Rikkonen ${ }^{3} \cdot$ Arja Erkkilä $^{3}$
}

Received: 23 April 2021 / Accepted: 6 December 2021 / Published online: 16 December 2021

(c) The Author(s) 2021

\begin{abstract}
Purpose The aim was to investigate the cross-sectional association of dietary omega-3 polyunsaturated fatty acids PUFA (alpha-linolenic acid (ALA), eicosapentaenoic acid (EPA), and docosahexaenoic acid (DHA)) intake with multiple physical functions, muscle mass and fat mass in older women.

Method Study subjects were 554 women from the Osteoporosis Risk Factor and Prevention Fracture Prevention Study, with dietary intake assessed with 3-day food record. Body composition was measured by dual-energy X-ray absorptiometry. Physical function measures included walking speed $10 \mathrm{~m}$, chair rises, one leg stance, knee extension, handgrip strength and squat. Short physical performance battery (SPPB) score was defined based on the European working group on sarcopenia criteria. Results The multivariable adjusted models showed statistically significant associations for dietary ALA with higher SPPB $(\beta=0.118, P=0.024)$, knee extension force at baseline $(\beta=0.075, P=0.037)$ and lower fat mass $(\beta=-0.081, P=0.034)$, as well as longer one-leg stance $(\beta=0.119, P=0.010)$, higher walking speed $(\beta=0.113, P=0.047)$, and ability to squat to the ground $(\beta=0.110, P=0.027)$ at baseline. Total dietary omega-3 PUFA was associated with better SPPB $(\beta=0.108$, $P=0.039)$, one-leg stance $(\beta=0.102, P=0.041)$ and ability to squat $(\beta=0.110, P=0.028)$, and with walking speed $(\beta=0.110, P=0.028)$. However, associations for dietary EPA and DHA with physical function and body composition were not significant.

Conclusion Dietary omega-3 and ALA, but not EPA and DHA, were positively associated with muscle strength and function in older women. The intake of omega-3 and its subtypes was not associated with muscle mass. Longitudinal studies are needed to show whether omega-3 intake may be important for muscle function in older women.
\end{abstract}

Keywords n-3 PUFA $\cdot$ Alpha-linolenic acid $\cdot$ Muscle mass $\cdot$ Physical function

\section{Introduction}

Masoud Isanejad

m.isanejad@liverpool.ac.uk

1 Department of Musculoskeletal and Ageing Sciences, Institute of Life Course and Medical Sciences, University of Liverpool, Liverpool L6 8TX, England, UK

2 Institute of Public Health and Clinical Nutrition, University of Eastern Finland, Kuopio Campus, Kuopio, Finland

3 Kuopio Musculoskeletal Research Unit, University of Eastern Finland, Kuopio Campus, Kuopio, Finland

4 Department of Obstetrics and Gynaecology, Kuopio, University Hospital, Kuopio, Finland
Ageing is associated with progressive physical function loss, changes in body composition by increase in fat mass and loss of muscle mass. These can lead to independence loss, frailty and consequently failing to carry on with activities of daily living, mental and cognitive decline [1]. Variety of assessment tools are currently available to asses physical function decline in older people, e.g. walking speed, hand grip strength, chair rises, knee extension force which are predictors of physical capacity, falls and hospitalization [2, $3]$, morbidity, and mortality [4, 5]. These physical function assessments are also highlighted as significant components of sarcopenia [6] and Fried frailty definition [7]. Similarly, 
muscle mass loss in older adults has been suggested as a clinical predictor of these adverse health outcomes $[8,9]$.

Investigating the role of diet and nutrients in preventing the onset of sarcopenia, deterioration of physical function and muscle mass loss are of high public health interest, mainly owing to the possibility of altering these factors via related interventions $[10,11]$. Among the components of a healthy diet, omega-3 PUFA are under focus in ageing research. The three main omega-3 PUFA are alpha-linolenic acid (ALA), eicosapentaenoic acid (EPA), and docosahexaenoic acid (DHA). Humans are unable to synthesize ALA, and as a result, it is essential to the diet. ALA is found mainly in plant oils such as flaxseed, soybean, and canola oils, whereas DHA and EPA are found in fish and other seafood [12]. These fatty acids and their food sources such as fish are embedded in public health recommendations for their beneficial health effects including anti-inflammatory properties [13], and they are suggested to have a role with multiple health outcomes [14] including muscle health [15, 16]. Noteworthy that WHO consortium on healthy ageing 2020 suggested that more research into role of omega-3 PUFA and healthy ageing are needed [17].

Muscle mass is maintained by a balance between muscle protein synthesis and breakdown. It has been proposed that n-3 PUFAs may be linked to muscle synthesis by enhancing the muscle protein synthesis or mTOR pathway. Finding from experimental studies, suggest that total EPA and DHA contributed to muscle protein synthesis and muscle anabolic stimulation [18]. In addition, in vivo studies have reported the changes in skeletal muscle phospholipid composition which can increase the amino acid uptake by these cells, triggering muscle anabolism $[18,19]$.

An important cause of loss of muscle mass and strength with ageing is low grade inflammation. A systematic review and meta-analysis by Tuttle et al., indicated that higher levels of circulating inflammatory cytokines are associated with lower skeletal muscle strength and muscle mass. Hence, a plausible pathway of dietary omega-3 PUFAs in prevention loss of muscle mass and strength can be reducing low grade inflammation [20-22] in older people.

Observational studies provided evidence on the positive association between plasma phospholipid PUFAs with knee extension and muscle mass [23], total omega-3, DHA, EPA and ALA intakes were positively associated with peak force (all individuals and men) in crude model in study by Rossato et al. (2020) [24], which did not remain significant in the adjusted model. A large cohort study also showed an indirect positive association for higher intake of fatty fish (rich source of omega-3) and higher in grip strength [25]. Most previous studies used single muscle strength outcomes, whereas the current study provides further evidence by studying the association for omega-3 PUFAs in relation to multiple physical function measures. In addition, the systematic review and meta-analysis indicated lack of sufficient data on omega-3 with muscle mass and strength [23]. The study hypothesis was that higher dietary ALA, EPA, DHA and total omega-3 PUFA intake associate with better clinical markers of muscle function and muscle mass and lower fart mass among older women aged $65-72$ years.

\section{Methods}

\section{Study design and participants}

Data for the present study were collected from the Osteoporosis Risk Factor and Prevention-Fracture Prevention Study (OSTPRE-FPS), which began in 2003 in Kuopio, Finland [24]. The OSTPRE-FPS was a randomized population-based open trial with a 3-year follow-up in 3,432 women (aged 66-71 years). The primary aim of the study was to determine whether vitamin D and calcium supplementation would be effective in preventing falls and fractures in postmenopausal women. For this sub study to investigate the effect of vitamin $\mathrm{D}$ and calcium supplementation on fracture prevention a sample of 750 women ( 375 from each of the intervention and control groups) was randomly selected from the 3432 women at the baseline underwent detailed examination at baseline and follow-up, including measurements of body composition, clinical, physical function, and laboratory tests. At the end of the trial $(n=593,79.0 \%), 306(40.8 \%)$ and 287 (38.2\%) subjects in the intervention and control groups of the subsample, respectively.

Only at the baseline 554 (93\%) women returned a valid food record at the baseline, and 39 women did not return the food record, or it was incomplete. Thus, for this cross-sectional study, the final analytical data included 554 women, with available dietary and physical function variables. The power analysis was performed based on the incidence of fractures [24]. There was no a priori power analysis to calculate the size of the subsample of 750 women randomly selected from the 3432 women at the baseline. All participants provided written permission for participation. The ethical committee of Kuopio University Hospital approved the study in October 2001. The study was registered at Clinical trials.gov by the identification NCT00592917.

\section{Dietary intake}

Dietary intake was assessed using 3-day food records. The records and instructions on completing the diary were sent to the subjects beforehand and they were asked to return the diaries on a research visit at the Kuopio Musculoskeletal Research Unit (KMRU) at the University of Kuopio. Subjects were advised to complete the diary information for 3 consecutive days, including 2 weekdays and either 
Saturday or Sunday. The types of fats used in bread, in cooking and baking were asked separately. Any unclarities in the diaries were checked by phone calls to the subjects by a nutritionist. Intake of different fatty acids and other nutrients from food records were calculated using the Nutrica program (version 2.5, Finnish Social Insurance Institute, Turku, Finland) [25].

\section{Body composition measurements}

Height and weight of participants were measured in light indoor clothing without shoes, body mass index (BMI) was calculated by weight $(\mathrm{kg})$ divided by height squared $(\mathrm{m})$. Fat mass and lean mass were measured by dual-energy X-ray absorptiometry (DXA) by specially trained nurses. The DXA measurements carried out using the same Lunar Prodigy adhering to the imaging and analysis protocols provided by the manufacturer (Lunar Co., Madison, WI, USA) [26]. DXA is currently a common tool suitable for estimation of body composition in terms of evaluating the ratio between fat, muscle, and bone in different parts of the body [27]. DXA also has been showed to be superior to bioimpedance for estimation of the body composition. Relative skeletal muscle index (RSMI) was calculated as the sum of the nonfat, non-bone skeletal muscle in arms and legs divided by height squared $\left(\mathrm{m}^{2}\right)$.

\section{Physical performance measurements}

Physical performance measures were assessed by trained nurses, including: hand grip strength $(\mathrm{kg})$, number of chair rises in $30 \mathrm{~s}$, ability to squat to ground, knee extension $(\mathrm{kg})$, walking speed $10 \mathrm{~m}(\mathrm{~m} / \mathrm{s})$ and tandem walk for $6 \mathrm{~m}(\mathrm{~m} / \mathrm{s})$, standing with closed eyes for $10 \mathrm{~s}$ and one leg stance performance for $30 \mathrm{~s}$; See Isanejad et al. 2016 [28] for details. Hand grip strength was measured in a controlled sitting position with a pneumatic hand-held dynamometer (Martin Vigorimeter, Germany) by calculating the mean of three successive measurements from the dominant hand.

\section{Short physical performance battery}

Short physical performance battery (SPPB) score was calculated using three individual measures of physical performance including walking speed $10 \mathrm{~m}(\mathrm{~m} / \mathrm{s})$, chair rises in $30 \mathrm{~s}$ and one leg stance performance categorized in quartiles [6], based on European Working Group on Sarcopenia definition [6] Each quartile was scored on scale of 1-4 points with the total score ranging to 12; higher scores of SPPB indicates better performance.

\section{Confounders}

Confounders were selected based on our prior work and literature reviews on dietary and physical function association [29], including age (years), energy intake (Kcal), protein intake $(\mathrm{g} / \mathrm{d})$, dietary vitamin $\mathrm{D}(\mu \mathrm{g} / \mathrm{d})$, dietary calcium $\left(\mathrm{mg} / \mathrm{d}\right.$ ), BMI $\left(\mathrm{kg} / \mathrm{m}^{2}\right.$ ) (or height for body composition analyses), hormone therapy (used, never used and current), smoking (smoker, nonsmokers), osteoporosis ( $\mathrm{T}$ and $\mathrm{Z}$ bone mineral density score for $2 \mathrm{SD}$ below the reference population), alcohol consumption (g/week) from a separate questionnaire, physical activity (hours/week) and intervention group. Details of computing the final physical activity variable has been published elsewhere [30]. Among the leisure and exercise variables the most common activities reported were skiing, walking, cycling, swimming, and aerobic exercise, explaining over $90 \%$ of the weekly physical activity, which was used to form the physical activity variable. The reported amount of weekly physical activity was used to form a long-term physical activity variable by summing up the average weekly physical activity at the baseline. History of diseases and medications data were collected by selfreported questionnaire at the baseline. Multimorbidity was defined as presence of two or more long-term health conditions, including depression, diabetes mellitus, hypertension, rheumatoid arthritis, coronary heart disease, osteoporosis, cancer, and pulmonary disease.

\section{Statistical analysis}

This study did not have inclusion or exclusion criteria and all women who had data on the PUFA dietary intake were included in this study. All statistical analyses were executed using SPSS software version 26 for Windows (IBM Corp., Armonk, NY). All tests were two-sided and a $P$ value of $<0.05$ was considered significant. Individual characteristics were analysed using ANOVA test for continuous variables and chi-square for categorical variables and presented for dietary quartiles of omega-3 PUFA and ALA. UNIANOVA controlled for selected confounders was used to account for variation in the dependent variables across the quartiles of omega-3 PUFA and ALA. Further, linear regression analyses calculated the standard coefficient $\beta$ controlled for selected confounders.

We tested the normality of overlay distribution we used the Kolmogrov and Sahapiro tests in the SPSS, and tests were not significant; and fit the normal curve.

Selected confounders for final analytical models were age, energy intake, protein intake, dietary vitamin D, dietary calcium, BMI (replaced with height for body composition analysis), multimorbidity, hormone therapy, smoking, alcohol consumption, physical activity per week, and osteoporosis. We also run a sensitivity analysis by including income per month 
as surrogate for socioeconomic status into the model, where results did not materially changed.

\section{Results}

The mean age was $67.8 \pm 1.8(n=554)$, mean BMI was $27.4 \mathrm{~kg} / \mathrm{m}^{2}$ and $43 \%$ of population had multimorbidity at the baseline. Analysis of baseline demographic and selected variables from self-reported questionnaires did not show significant differences according to quartiles of dietary omega-3 PUFA and ALA (Table 1). Among dietary intake variables higher omega-3 PUFA was associated with lower dietary calcium and higher vitamin D intake (Table 1). Higher ALA intake was associated with lower protein intake (g/day), calcium intake (mg/day) and vitamin D intake ( $\mu \mathrm{g} /$ day). Table 2 presents results for the dietary omega-3 intakes, alpha-linolenic acid (g/day) $1.4(0.7)$, eicosapentaenoic acid ( $\mathrm{g} /$ day) 0.12 (0.1), docosahexaenoic acid (g/day) $0.28(0.3)$.

Table 3 presents the association of physical function and body composition for omega-3 PUFA and ALA quartiles. After controlling for selected confounders, higher quartile of omega-3 PUFA and ALA were associated with higher SPBB score ( $P=0.020$ and $P=0.010$, respectively), longer one leg stance ( $P=0.050$ and $P=0.035$, respectively), faster walking speed $10 \mathrm{~m}(P=0.048$ and $P=0.005$, respectively), and lower frequency of women with inability to squat to the ground ( $P=0.014$ and $P=0.004$, respectively). Additionally, higher quartile for ALA was associated with lower fat mass $(P=0.001)$, and higher grip strength $(P=0.047)$.

Table 4 shows results for the linear regression analysis controlling for selected confounders (age, energy intake, protein intake, dietary vitamin $\mathrm{D}$, dietary calcium, BMI, hormone therapy, smoking, osteoporosis, alcohol consumption, physical activity hour/per week and intervention group). Both omega-3 PUFA and ALA were positively and significantly associated with SPPB $(\beta=0.108, P=0.039$ and $\beta=0.118, P=0.034$, respectively), one leg stance $(\beta=0.102$, $P=0.041$ and $\beta=0.119, P=0.010$, respectively), walking speed $(\beta=0.110, P=0.028$ and $\beta=0.113, P=0.047$, respectively), and with lower inability to squat to ground ( $\beta=0.110, P=0.028$ and $\beta=0.112, P=0.027$, respectively). Also, ALA intake was positively associated with knee extension force $(\beta=0.075, P=0.037)$ and inversely associated with fat mass $(\beta=-0.081, P=0.034)$. Similar associations were observed using 1-SD increment of omega-3 PUFA, ALA, EPA and DHA dietary intake (data not shown).

\section{Discussion}

The main result of the present cross-sectional study was that dietary intake of ALA and total omega-3 PUFA were positively associated with physical function assessments, including faster walking speed $10 \mathrm{~m}$, better performance at one leg stance, ability to squat to the ground and SPPB. Higher ALA quartile was also associated with greater grip strength and lower fat mass. Findings showed no statistically significant association for dietary EPA and DHA with physical function assessments.

Although previous studies reported association between omega-3 PUFA intake with walking speed [31, 32], peak force and knee extension [33]. Robinson et al. found that fatty fish intake was the largest predictor of hand grip strength [34]. Although not directly this suggests that omega-3 PUFA may at least partially explain this association. In a cross-sectional study among French communitydwelling older adults, a higher proportion of the omega-3 PUFAs EPA and DHA in plasma was associated with a higher gait speed [35]. Similar cross-sectional result was reported among 1273 older adults involved in the InCHIANTI study, significant positive association between omega-3 PUFA with the faster completion of a 7-m walking test [36]. Recent study by Rossato et al. [37] showed that the intake of total omega-3 PUFA was positively associated with peak force in older men but not in women. The present study complemented the previous findings in only women, showing where positive association for total omega-3 and ALA was detected with a wider range of physical function outcomes including positive association with one leg stance, ability to squat and grip strength and SPPB. In contrary, the study by Fougere [31] among 400 participants aged $75.2( \pm 4.3)$ years, reported negative association between high levels of baseline red blood cell omega-3 PUFAs and SPPB, the reason for this negative findings was partially explained by the ceiling effect as the mean SPPB was considered particularly high (10.7 out of 12) and a narrow range could reduce that statistical power, whereas in present study SPPB mean was 7.7 out of 12 .

Robust assessments indicated in the RCTs for direct effects omega-3 PUFAs on muscle mass and function in the older adult population are scarce. Findings of study by Smith et al. showed that six months omega-3 PUFA therapy in sixty healthy 60- to 85-year-old men and women, increased thigh muscle volume, and handgrip strength, and tended to increase average isokinetic power compared to control group (receiving corn oil) [38]. Another RCT of omega 3 fatty acids supplementation showed no statistically significant differences either in muscle mass or in the hand grip and time up and go tests compared to control group [39]. Identifying the seldom effect of EPA and DHA on muscle mass and strength in the elderly is not feasible based on limited studies, however, a recent meta-analysis of randomized controlled trials (RCTs) found that supplementation with both types of n-3 PUFAs (EPA and/ or DHA) elicits a $\sim 0.33 \mathrm{~kg}$ increase in muscle mass. In addition, EPA and DHA have positive effect on the muscle 


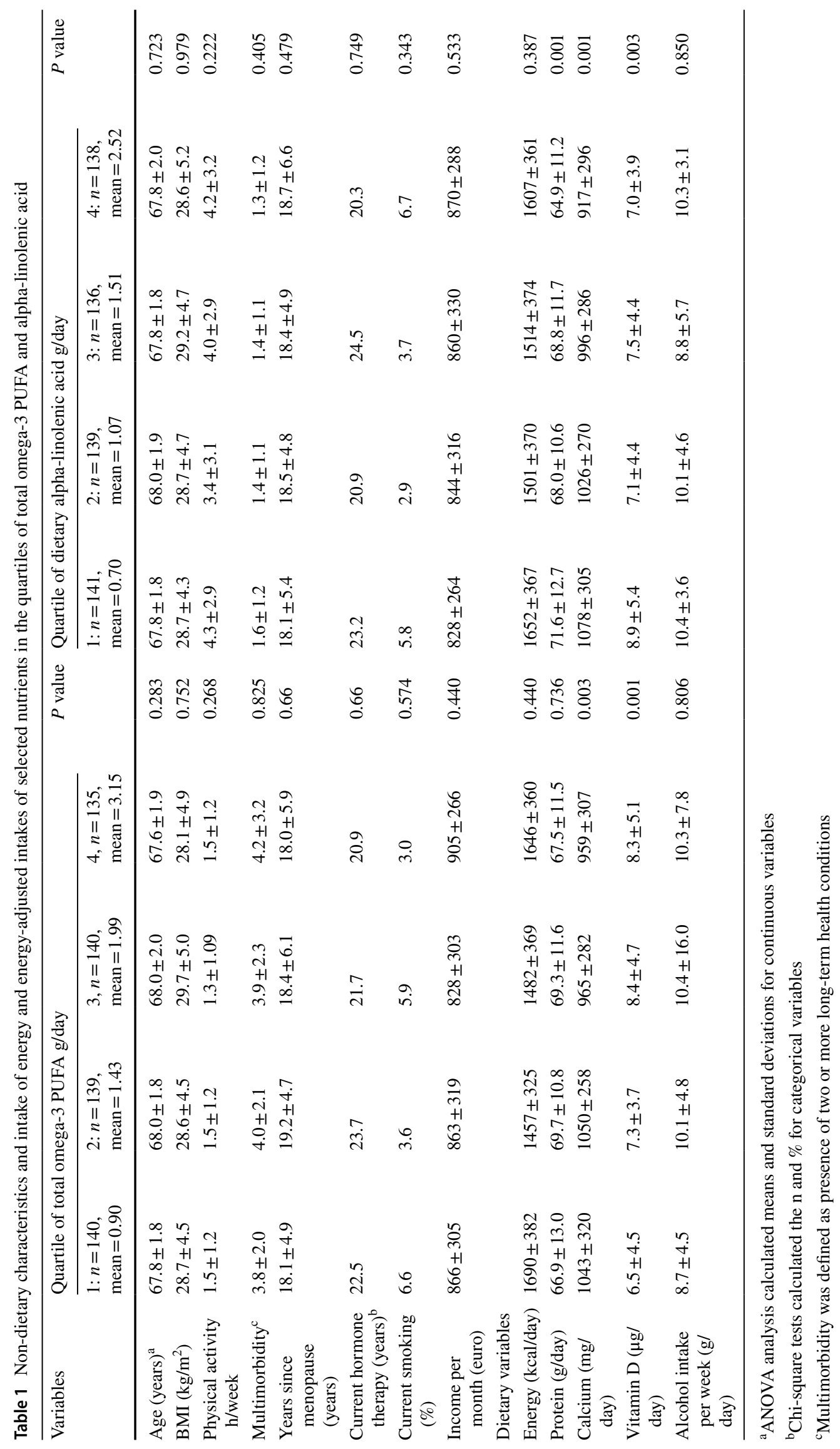


Table 2 Dietary omega 3 PUFAs intake

\begin{tabular}{lc}
\hline Dietary omega 3 intake & Means \pm SD $(n=554)$ \\
\hline Alpha-linolenic acid (g/day) & $1.4 \pm 0.7$ \\
Eicosapentaenoic acid (g/day) & $0.12 \pm 0.1$ \\
Docosahexaenoic acid (g/day) & $0.28 \pm 0.3$ \\
\hline
\end{tabular}

protein synthesis or reducing oxidative stress in animal models $[40,41]$.

Currently lower extremity functions are the main indicator for mobility as a clinical screening tool [42], and it maybe that by ageing lower extremity function are more prone to change and perhaps influenced by external stimuli such as diet and exercise. ALA is a plant-derived n-3 fatty acid that mainly exists in flaxseed, soybean, perilla, walnut, and canola oils. In healthy adults, only $5-10 \%$ and $2-5 \%$ of ALA can be converted into EPA and DHA, respectively [43]. Also, the conversion of ALA to EPA and DHA in men is estimated to be as low as $<8 \%$ and $<4 \%$ respectively, whilst in women it is slightly higher at 21 and $9 \%$, respectively [44]. Therefore, inter-individual variability should be considered in future studies and possible EPA and DHA supplementation.

Our study population is characterized by a high intake of omega-3 PUFA and ALA. The analysis of 3-day food record in this study showed that OSTPRE-FPS older women consumed EPA + DHA $0.41 \pm 0.47$ (g/day), ALA $1.4 \pm 0.8 \mathrm{~g} /$ day (corresponding to $0.83 \%$ of the mean energy intake), and total omega-3 PUFA $8.8 \pm 3.4 \mathrm{~g} /$ day, which all are within the range of recommendation by Nordic Nutrition recommendation 2012: minimum $1 \mathrm{E} \%$ from $\mathrm{n}-3$ fatty acids and intake of $\mathrm{EPA}+\mathrm{DHA}$ up to $200-250 \mathrm{mg} /$ day.

There are multiple potential mechanisms which can explain the observed association in this study. Low grade inflammation has been considered as important trigger for inflammageing occurring with aging, which is a chronic state of slightly increased plasma levels of pro-inflammatory mediators, such as tumor necrosis factor $\alpha$ (TNF- $\alpha$ ), interleukin 6 (IL-6) and C-reactive protein (CRP) [45]. Although the causative link between inflammatory markers and the risk for functional decline and mortality in elderly are hard to established, strong correlation between these two have been identified in previous studies [46]. Without pronouncing the causal links between inflammatory mediator and physical function, the current literature suggests that nutritional factors and particularly omega-3 PUFA are among the biological mechanism to reduce low grade inflammation which can play role to delay or prevent the onset of physical function decline $[16,47]$. Results from a study indicated that ALA decreases the plasma level of inflammatory cytokines (TNFalpha and IL-6) in the elderly [40]. In addition, findings from experimental studies, although limited, suggest that omega-3

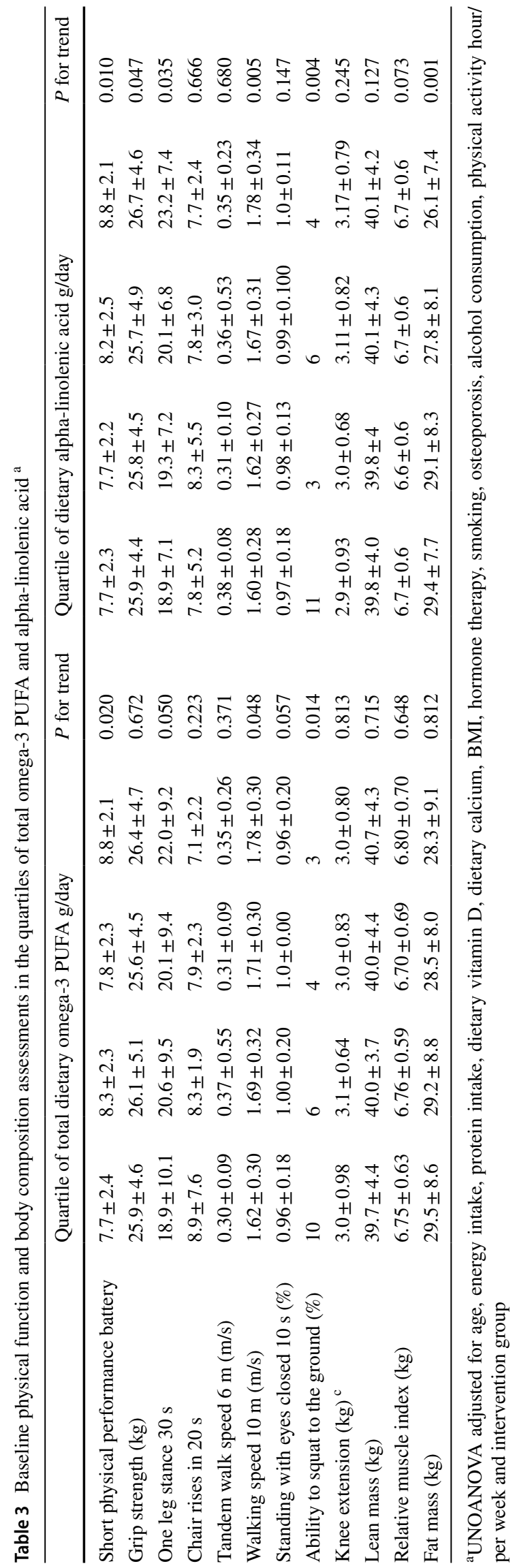


Table 4 Cross-sectional association of dietary omega-3 PUFA and physical function and body composition. PUFA, polyunsaturated fatty acid; add EPA, DHA and ALA

\begin{tabular}{|c|c|c|c|c|c|c|c|c|}
\hline & \multicolumn{2}{|c|}{ Total omega-3 PUFA } & \multicolumn{2}{|l|}{ EPA } & \multicolumn{2}{|l|}{ DHA } & \multicolumn{2}{|c|}{ Alpha-linolenic acid } \\
\hline & $\begin{array}{l}\text { Standardized } \\
\text { coefficient } \beta\end{array}$ & $P^{\mathrm{a}}$ & $\begin{array}{l}\text { Standardized } \\
\text { coefficient } \beta\end{array}$ & $P^{\mathrm{a}}$ & $\begin{array}{l}\text { Standardized } \\
\text { coefficient } \beta\end{array}$ & $P^{\mathrm{a}}$ & $\begin{array}{l}\text { Standardized } \\
\text { coefficient } \beta\end{array}$ & $P^{\mathrm{a}}$ \\
\hline Short physical performance battery & 0.108 & 0.039 & 0.015 & 0.752 & 0.013 & 0.752 & 0.118 & 0.024 \\
\hline Grip strength (kg) & 0.018 & 0.679 & 0.022 & 0.983 & 0.024 & 0.592 & 0.013 & 0.578 \\
\hline One leg stance $30 \mathrm{~s}$ & 0.102 & 0.041 & 0.001 & 0.730 & 0.008 & 0.850 & 0.119 & 0.010 \\
\hline Chair rises in $20 \mathrm{~s}$ & -0.043 & 0.329 & -0.286 & 0.775 & -0.013 & 0.487 & -0.014 & 0.348 \\
\hline Tandem walk speed $6 \mathrm{~m}(\mathrm{~m} / \mathrm{s})$ & 0.035 & 0.451 & -0.017 & 0.715 & -0.030 & 0.411 & 0.058 & 0.216 \\
\hline Walking speed $10 \mathrm{~m}(\mathrm{~m} / \mathrm{s})$ & 0.110 & 0.028 & 0.028 & 0.507 & 0.026 & 0.537 & 0.113 & 0.047 \\
\hline Standing with eyes closed $10 \mathrm{~s}(\%)$ & -0.017 & 0.696 & -0.039 & 0.377 & -0.038 & 0.387 & 0.003 & 0.943 \\
\hline Ability to squat to the ground (\%) & 0.110 & 0.028 & 0.025 & 0.545 & 0.034 & 0.410 & 0.112 & 0.027 \\
\hline Knee extension $(\mathrm{kg})^{\mathrm{c}}$ & 0.039 & 0.394 & -0.096 & 0.039 & -0.104 & 0.075 & 0.096 & 0.037 \\
\hline Lean mass $(\mathrm{kg})$ & -0.001 & 0.976 & -0.029 & 0.471 & 0.048 & 0.158 & 0.030 & 0.372 \\
\hline Relative muscle index (kg) & 0.009 & 0.832 & 0.004 & 0913 & 0.039 & 0.345 & 0.017 & 0.684 \\
\hline Fat mass (kg) & 0.001 & 0.970 & 0.001 & 0.979 & 0.013 & 0.740 & -0.081 & 0.034 \\
\hline
\end{tabular}

${ }^{a}$ Adjusted for age, energy intake, protein intake, dietary vitamin D, dietary calcium, BMI, hormone therapy, smoking, osteoporosis, alcohol consumption, physical activity hour/per week and intervention group

PUFA contribute to muscle protein synthesis anabolic pathway. This finding was consistent with the animal models and cell culture suggesting that omega-3 PUFA can enhance the muscle protein synthesis [48]. Current evidence also suggest that omega-3 PUFAs are potentially able to enhance the capacity of muscle cells for more permeable reaction with regard to necessary nutrients, such as glucose and amino acids [8]. The finding of this study on positive association for omega-3 PUFA, and ALA with physical function outcomes can be explained by these underlying mechanisms, however, further studies on effect of omega-3 PUFAon muscle function and muscle protein synthesis are required. Besides, the positive finding may be explained by the indirect relationship of omega-3 PUFA as they can improve blood lipid profile and heart function [49] which in turn are associated with healthy ageing $[40,50]$. Previously, we have published that Mediterranean and Baltic Sea diets have positive associations with improved physical function and lean mass in this data [29]. We acknowledge that only for ALA the correlation coefficients relating to Mediterranean $\operatorname{diet}(r=0.210, P<0.001)$ and Baltic sea diet were statistically significant $(r=0.126, P=0.004)$, but not with EPA or DHA. Even though we cannot exclude other confounding factors it maybe suggesting that role of fatty acids should be considered more in the whole dietary approach rather than single nutrients which may explain the inconsistent results of previous studies.

There are possible limitations to consider. We conducted secondary analysis on data from a RCT and the RCT was not originally designed to investigate the associations reported here from the baseline of the study. Our study population included only relatively healthy older women from a homogenous Finnish population, thus results may not be generalized to other older populations. We controlled for multiple confounders; the probability of other lifestyle factors related to higher omega-3 PUFA intake affecting physical functions cannot be excluded. Finally, since the nature of this study is observational and using self-assessment tools such as food diary as the data source, it might be that dietary intake including omega-3 PUFA can be over or underestimated.

\section{Conclusion}

In conclusion, this study in older women suggests that higher omega-3 PUFA and ALA s were positively associated with measures of physical capacity (walking speed $10 \mathrm{~m}$, at one leg stance, ability to squat to the ground and SPPB and grip strength) but not with the muscle mass. However, there is considerable scope for future work to fully elucidate the potential of omega-3 PUFA in muscle function as well as on possible gender differences of omega- 3 function in men and women.

Acknowledgements The authors would like to extend their appreciation to OSTPRE investigator and participants.

Author contributions $\mathrm{MI}$ and BT designed the study and performed the statistical analysis, where MI has the primary responsibility for the data analysis. MI, TR, and AE wrote the paper in collaboration. BT, TR, JS, HK, and AM review the manuscript for it's scientific and intellectual content. 
Funding Dr. Masoud Isanejad received general research grants from the Finnish Cultural Foundation.

\section{Declarations}

Conflict of interest The authors declare no conflicts of interest.

Ethical approval All participants provided written permission for participation. The ethical committee of Kuopio University Hospital approved the study in October 2001. The study was registered at Clinical trials.gov by the identification NCT00592917.

Open Access This article is licensed under a Creative Commons Attribution 4.0 International License, which permits use, sharing, adaptation, distribution and reproduction in any medium or format, as long as you give appropriate credit to the original author(s) and the source, provide a link to the Creative Commons licence, and indicate if changes were made. The images or other third party material in this article are included in the article's Creative Commons licence, unless indicated otherwise in a credit line to the material. If material is not included in the article's Creative Commons licence and your intended use is not permitted by statutory regulation or exceeds the permitted use, you will need to obtain permission directly from the copyright holder. To view a copy of this licence, visit http://creativecommons.org/licenses/by/4.0/.

\section{References}

1. Yi C, Lin J, Cao P, Chen J, Zhou T, Yang R, Lu S, Yu X, Yang X (2018) Prevalence and prognosis of coexisting frailty and cognitive impairment in patients on continuous ambulatory peritoneal dialysis. Sci Rep 8(1):1-10

2. Yang M, Liu Y, Zuo Y, Tang H (2019) Sarcopenia for predicting falls and hospitalization in community-dwelling older adults: EWGSOP versus EWGSOP2. Sci Rep 9(1):1-8

3. Bohannon RW (2019) Grip strength: an indispensable biomarker for older adults. Clin Interv Aging 14:1681

4. Sayer AA, Kirkwood TB (2015) Grip strength and mortality: a biomarker of ageing? Lancet (London, England) 386(9990):226

5. Elbaz A, Sabia S, Brunner E, Shipley M, Marmot M, Kivimaki M, Singh-Manoux A (2013) Association of walking speed in late midlife with mortality: results from the Whitehall II cohort study. Age 35(3):943-952

6. Cruz-Jentoft AJ, Bahat G, Bauer J, Boirie Y, Bruyère O, Cederholm T, Cooper C, Landi F, Rolland Y, Sayer AA (2019) Sarcopenia: revised European consensus on definition and diagnosis. Age Ageing 48(1):16-31

7. Fried LP, Tangen CM, Walston J, Newman AB, Hirsch C, Gottdiener J, Seeman T, Tracy R, Kop WJ, Burke G, McBurnie MA, Cardiovascular Health Study Collaborative Research G (2001) Frailty in older adults: evidence for a phenotype. J Gerontol Ser Biol Sci Med Sci 56(3):146

8. Miotto PM, McGlory C, Bahniwal R, Kamal M, Phillips SM, Holloway GP (2019) Supplementation with dietary $\omega$-3 mitigates immobilization-induced reductions in skeletal muscle mitochondrial respiration in young women. FASEB J 33(7):8232-8240

9. Lunt E, Ong T, Gordon AL, Greenhaff PL, Gladman JRF (2021) The clinical usefulness of muscle mass and strength measures in older people: a systematic review. Age Ageing 50(1):88-95. https://doi.org/10.1093/ageing/afaa123

10. Veronese N, Stubbs B, Punzi L, Soysal P, Incalzi RA, Saller A, Maggi S (2019) Effect of nutritional supplementations on physical performance and muscle strength parameters in older people: a systematic review and meta-analysis. Ageing Res Rev 51:48-54

11. Cruz-Jentoft AJ, Woo J (2019) Nutritional interventions to prevent and treat frailty. Curr Opin Clin Nutr Metab Care 22(3):191-195

12. Calder PC (2015) Marine omega-3 fatty acids and inflammatory processes: effects, mechanisms and clinical relevance. Biochimica et Biophysica (BBA) Acta Mol Cell Biol Lipids 1851(4):469-484

13. Di Girolamo FG, Situlin R, Mazzucco S, Valentini R, Toigo G, Biolo G (2014) Omega-3 fatty acids and protein metabolism: enhancement of anabolic interventions for sarcopenia. Curr Opin Clin Nutr Metab Care 17(2):145-150. https://doi.org/10.1097/ MCO.0000000000000032

14. Abdelhamid AS, Brown TJ, Brainard JS, Biswas P, Thorpe GC, Moore HJ, Deane KHO, Summerbell CD, Worthington HV, Song F (2020) Omega-3 fatty acids for the primary and secondary prevention of cardiovascular disease. Cochrane Database Syst Rev 3(3):CD003177. https://doi.org/10.1002/14651858.CD003177. pub5

15. Troesch B, Eggersdorfer M, Laviano A, Rolland Y, Smith AD, Warnke I, Weimann A, Calder PC (2020) Expert opinion on benefits of long-chain omega-3 fatty acids (DHA and EPA) in aging and clinical nutrition. Nutrients 12(9):2555

16. Huang Y-H, Chiu W-C, Hsu Y-P, Lo Y-L, Wang Y-H (2020) Effects of omega-3 fatty acids on muscle mass, muscle strength and muscle performance among the elderly: a meta-analysis. Nutrients 12(12):3739

17. World Health O (2020) WHO clinical consortium on healthy ageing 2019: report of consortium meeting held 21-22 November 2019, Geneva, Switzerland

18. Smith GI, Atherton P, Reeds DN, Mohammed BS, Rankin D, Rennie MJ, Mittendorfer B (2011) Dietary omega-3 fatty acid supplementation increases the rate of muscle protein synthesis in older adults: a randomized controlled trial. Am J Clin Nutr 93(2):402-412

19. Stillwell W, Wassall SR (2003) Docosahexaenoic acid: membrane properties of a unique fatty acid. Chem Phys Lipid 126(1):1-27

20. Boreskie KF, Oldfield CJ, Hay JL, Moffatt TL, Hiebert BM, Arora RC, Duhamel TA (2020) Myokines as biomarkers of frailty and cardiovascular disease risk in females. Exp Gerontol 133:110859

21. Calder PC, Bosco N, Bourdet-Sicard R, Capuron L, Delzenne N, Doré J, Franceschi C, Lehtinen MJ, Recker T, Salvioli S (2017) Health relevance of the modification of low grade inflammation in ageing (inflammageing) and the role of nutrition. Ageing Res Rev 40:95-119

22. Franceschi $\mathrm{C}$, Bonafè $\mathrm{M}$, Valensin S, Olivieri F, De Luca M, Ottaviani E, De Benedictis G (2000) Inflamm-aging: an evolutionary perspective on immunosenescence. Ann N Y Acad Sci 908(1):244-254

23. Rossato LT, Schoenfeld BJ, de Oliveira EP (2020) Is there sufficient evidence to supplement omega- 3 fatty acids to increase muscle mass and strength in young and older adults? Clin Nutr 39(1):23-32

24. Kärkkäinen M, Tuppurainen M, Salovaara K, Sandini L, Rikkonen T, Sirola J, Honkanen R, Jurvelin J, Alhava E, Kröger $\mathrm{H}$ (2010) Effect of calcium and vitamin D supplementation on bone mineral density in women aged 65-71 years: a 3-year randomized population-based trial (OSTPRE-FPS). Osteoporos Int 21(12):2047-2055. https://doi.org/10.1007/s00198-009-1167-8

25. Jarvinen R, Tuppurainen M, Erkkilä AT, Penttinen P, Karkkainen M, Salovaara K, Jurvelin JS, Kroger H (2012) Associations of dietary polyunsaturated fatty acids with bone mineral density in elderly women. Eur J Clin Nutr 66(4):496-503. https://doi.org/10. 1038/ejcn.2011.188

26. Sjoblom S, Suuronen J, Rikkonen T, Honkanen R, Kroger H, Sirola J (2013) Relationship between postmenopausal 
osteoporosis and the components of clinical sarcopenia. Maturitas 75(2):175-180. https://doi.org/10.1016/j.maturitas.2013.03.016

27. Lohman M, Tallroth K, Kettunen JA, Marttinen MT (2009) Reproducibility of dual-energy X-ray absorptiometry total and regional body composition measurements using different scanning positions and definitions of regions. Metab Clin Exp 58(11):16631668. https://doi.org/10.1016/j.metabol.2009.05.023

28. Isanejad M, Mursu J, Sirola J, Kröger H, Rikkonen T, Tuppurainen M, Erkkilä AT (2016) Dietary protein intake is associated with better physical function and muscle strength among elderly women. Br J Nutr 115(7):1281-1291

29. Isanejad M, Sirola J, Mursu J, Rikkonen T, Kröger H, Tuppurainen M, Erkkilä AT (2018) Association of the Baltic Sea and Mediterranean diets with indices of sarcopenia in elderly women, OSPTRE-FPS study. Eur J Nutr 57(4):1435-1448

30. Sjöblom S, Sirola J, Rikkonen T, Erkkilä AT, Kröger H, Qazi SL, Isanejad M (2020) Interaction of recommended levels of physical activity and protein intake is associated with greater physical function and lower fat mass in older women: Kuopio Osteoporosis Risk Factor-(OSTPRE) and Fracture-Prevention Study. Br J Nutr 123(7):826-839

31. Fougère $B$, de Souto BP, Goisser $S$, Soriano G, Guyonnet $S$, Andrieu S, Vellas B, Carrié I, Brigitte L, Faisant C (2018) Red blood cell membrane omega-3 fatty acid levels and physical performance: cross-sectional data from the MAPT study. Clin Nutr 37(4):1141-1144

32. Fougère $B$, Goisser $S$, Cantet $C$, Soriano G, Guyonnet $S$, Barreto PDS, Cesari M, Andrieu S, Vellas B (2017) Omega-3 fatty acid levels in red blood cell membranes and physical decline over 3 years: longitudinal data from the MAPT study. Geroscience 39(4):429-437

33. Reinders I, Song X, Visser M, Eiriksdottir G, Gudnason V, Sigurdsson S, Aspelund T, Siggeirsdottir K, Brouwer IA, Harris TB, Murphy RA (2014) Plasma phospholipid PUFAs are associated with greater muscle and knee extension strength but not with changes in muscle parameters in older adults. J Nutr 145(1):105112. https://doi.org/10.3945/jn.114.200337

34. Robinson SM, Jameson KA, Batelaan SF, Martin HJ, Syddall HE, Dennison EM, Cooper C, Sayer AA, Hertfordshire Cohort Study G (2008) Diet and its relationship with grip strength in community-dwelling older men and women: the Hertfordshire cohort study. J Am Geriatr Soc 56(1):84-90

35. Frison E, Boirie Y, Peuchant E, Tabue-Teguo M, BarbergerGateau P, Féart C (2017) Plasma fatty acid biomarkers are associated with gait speed in community-dwelling older adults: the Three-City-Bordeaux study. Clin Nutr 36(2):416-422

36. Abbatecola AM, Cherubini A, Guralnik JM, Lacueva CA, Ruggiero C, Maggio M, Bandinelli S, Paolisso G, Ferrucci L (2009) Plasma polyunsaturated fatty acids and age-related physical performance decline. Rejuvenation Res 12(1):25-32

37. Rossato LT, de Branco FMS, Azeredo CM, Rinaldi AEM, de Oliveira EP (2020) Association between omega-3 fatty acids intake and muscle strength in older adults: a study from National Health and Nutrition Examination Survey (NHANES) 1999-2002. Clin Nutr 39(11):3434-3441. https://doi.org/10.1016/j.clnu.2020. 03.001
38. Smith GI, Julliand S, Reeds DN, Sinacore DR, Klein S, Mittendorfer B (2015) Fish oil-derived n-3 PUFA therapy increases muscle mass and function in healthy older adults. Am J Clin Nutr 102(1):115-122

39. Krzymińska-Siemaszko R, Czepulis N, Lewandowicz M, Zasadzka E, Suwalska A, Witowski J, Wieczorowska-Tobis K (2015) The effect of a 12-week omega-3 supplementation on body composition, muscle strength and physical performance in elderly individuals with decreased muscle mass. Int J Environ Res Public Health 12(9):10558-10574

40. Gray SR, Mittendorfer B (2018) Fish oil-derived n-3 polyunsaturated fatty acids for the prevention and treatment of sarcopenia. Curr Opin Clin Nutr Metab Care 21(2):104-109

41. Tan A, Sullenbarger B, Prakash R, McDaniel JC (2018) Supplementation with eicosapentaenoic acid and docosahexaenoic acid reduces high levels of circulating proinflammatory cytokines in aging adults: a randomized, controlled study. Prostaglandins Leukot Essent Fatty Acids 132:23-29

42. Edmunds K, Gíslason M, Sigurðsson S, Guðnason V, Harris T, Carraro U, Gargiulo P (2018) Advanced quantitative methods in correlating sarcopenic muscle degeneration with lower extremity function biometrics and comorbidities. PLoS ONE 13(3):e0193241

43. Wijendran V, Hayes KC (2004) Dietary n-6 and n-3 fatty acid balance and cardiovascular health. Annu Rev Nutr 24:597-615

44. Burdge GC, Calder PC (2005) Conversion of $\alpha$-linolenic acid to longer-chain polyunsaturated fatty acids in human adults. Reprod Nutr Dev 45(5):581-597

45. Ferrucci L, Fabbri E (2018) Inflammageing: chronic inflammation in ageing, cardiovascular disease, and frailty. Nat Rev Cardiol 15(9):505

46. Pavasini R, Guralnik J, Brown JC, Di Bari M, Cesari M, Landi F, Vaes B, Legrand D, Verghese J, Wang C (2016) Short physical performance battery and all-cause mortality: systematic review and meta-analysis. BMC Med 14(1):1-9

47. Yan Y, Jiang W, Spinetti T, Tardivel A, Castillo R, Bourquin C, Guarda G, Tian Z, Tschopp J, Zhou R (2013) Omega-3 fatty acids prevent inflammation and metabolic disorder through inhibition of NLRP3 inflammasome activation. Immunity 38(6):1154-1163

48. Kamolrat T, Gray SR (2013) The effect of eicosapentaenoic and docosahexaenoic acid on protein synthesis and breakdown in murine $\mathrm{C} 2 \mathrm{C} 12$ myotubes. Biochem Biophys Res Commun 432(4):593-598

49. Alexander DD, Miller PE, Van Elswyk ME, Kuratko CN, Bylsma LC (2017) A meta-analysis of randomized controlled trials and prospective cohort studies of eicosapentaenoic and docosahexaenoic long-chain omega- 3 fatty acids and coronary heart disease risk. In: Mayo Clinic Proceedings, 2017. Vol conference Proceedings. Elsevier, pp 15-29

50. Ruxton CHS, Derbyshire E, Toribio-Mateas M (2016) Role of fatty acids and micronutrients in healthy ageing: a systematic review of randomised controlled trials set in the context of European dietary surveys of older adults. J Hum Nutr Diet 29(3):308-324 